\title{
Netherton syndrome previously misdiagnosed as hyper IgE syndrome caused by a probable mutation in SPINK5 C
}

\author{
Kemal Özyurt ${ }^{1}$, Mustafa Atasoy ${ }^{1}$, Ragıp Ertaş ${ }^{1}$, Yılmaz Ulaş ${ }^{1}$, Muhammed Reşat Akkuş ${ }^{1}$, \\ Aslihan Kiraz ${ }^{2}$, Hans Christian Hennies ${ }^{3,4,5}$ \\ ${ }^{1}$ Dermatology Clinic and ${ }^{2}$ Medical Genetics Health Science University, Kayseri Training and Research Center, Kayseri, \\ Turkey; ${ }^{3}$ Dermatogenetics, Department of Biological Sciences, University of Huddersfield, Huddersfield, UK; ${ }^{4}$ Cologne \\ Center for Genomics and ${ }^{5}$ Cluster of Excellence for Aging Research, University of Cologne University of Cologne, Cologne, \\ Germany.E-mail:drkozyurt@gmail.com \\ Received: 20th February 2018, Revised: 21st September 2018, Accepted: 24th September 2018
}

SUMMARY: Özyurt K, Atasoy M, Ertaş R, Ulaş Y, Akkuş MR, Kiraz A, Hennies HC. Netherton syndrome previously misdiagnosed as hyper IgE syndrome caused by a probable mutation in SPINK5 C. Turk J Pediatr 2019; 61: 604-607.

Netherton syndrome (NS, MIM256500) is an autosomal recessive disorder that includes ichthyosis linearis circumflexa and a predisposition to allergies, asthma, and eczema, with hypereosinophilia, trichorrhexis invaginata, and elevated serum IgE levels. The genetic bases of Netherton syndrome are mutations in the gene SPINK5, and the Lymphoepitheial Kazal type related inhibitor, a serine protease inhibitor, is encoded by SPINK.

Here a case is presented which showed a probable splice site mutation in SPINK5, which was previously unknown in databases and the literature, to point out the misdiagnosis of Hyper IgE Syndrome in the early presentation of the phenotype. This case highlights that a genetic test can be critical for identifying NS. The finding of underlying mutations contributes to the understanding of Netherton syndrome and is instrumental in indicating a specific therapy. Notably, treatment with acitretin has significantly improved both the ichthyosis linearis circumflexa and eczema in our patient.

Key words: Netherton syndrome, eczema, hyper IgE syndrome, serum IgE, ichthyosis.

Netherton syndrome (NS, MIM256500) is an autosomal recessive disorder that includes ichthyosis linearis circumflexa (ILC) and a predisposition to allergies, asthma, and eczema, with hypereosinophilia, trichorrhexis invaginata, and elevated serum IgE levels. ${ }^{1}$ Severe eczema and elevated serum IgE are common in various genodermatoses. ${ }^{2,3}$ In complicated cases, a genetic test can be critical for identifying NS. The genetic bases of NS are mutations in the gene SPINK5, and the Lymphoepitheial Kazal type related inhibitor, a serine protease inhibitor, is encoded by SPINK. ${ }^{1}$
We think it is valuable to share this case, which showed a probable splice site mutation in SPINK5, which was previously unknown in databases and the literature, to point out the misdiagnosis of Hyper IgE Syndrome (HIS) in the early presentation of the phenotype.

\section{Case Report}

A four-year-old male presented to the dermatology clinic. He was the son of consanguineous parents (first cousins) who showed no signs of skin disease. His parents described the boy as having uneasiness, 
irritability, intensive scratching, and red scaly patches all over his body and scalp. He was given intravenous IgG therapy because of his diagnosis of HIES for one year, with no benefit seen.

Collodion membrane and congenital erythroderma were recorded in his medical files at birth (Figs. 1a and 1b). Numerous consultations and laboratory analyses from birth to the present are summarized in Table I. He experienced attacks of urticaria/ angioedema and elevated serum IgE levels in his subsequent life. He had no other recurrent infections.

A red-purple patch was observed on the right half of his face, beginning from the right upper lip and extending to the upper part of the right cheek to the right temple. This was diagnosed as capillary malformation. Erythematous and scaly patches were seen on the forehead, left arm, shoulder, back, trunk, upper chest, and thighs, respectively (Figs. 2a, $2 \mathrm{~b}$, and $3 \mathrm{a}$ ). These lesions were diagnosed as ILC. Histopathological examination revealed psoriasiform dermatitis compatible with ILC. We did not observe any pathology on the patient's hair.

Genetic analysis was performed on a DNA sample from the patient to evaluate possible mutations. It is an important issue to evaluate the origin to differentiate whether this is de novo or parental. However, we were unable to make a family analysis because all family

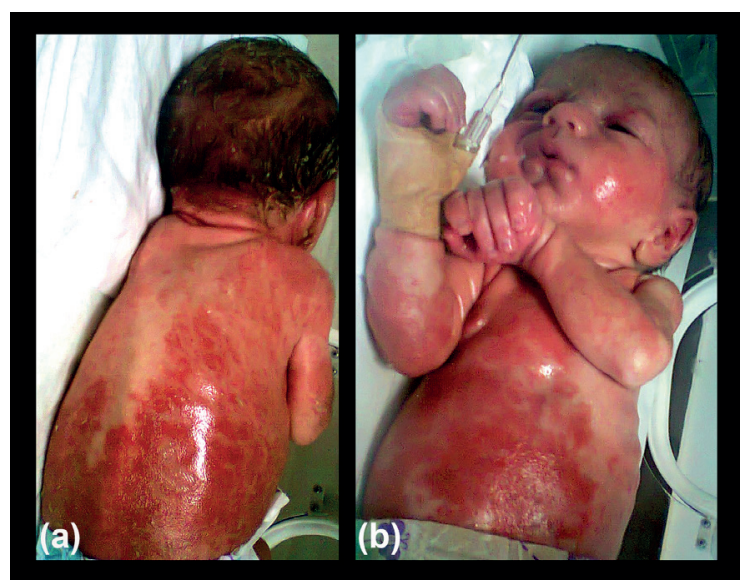

Figs. 1a and 1b. Diffuse erythematous and scaly patches on the body and scalp during the patient's neonatal period.
Table I. Clinical and Laboratory Summaries of the Patient from Birth.

Birth to age 1

E\%: 14.5, IgE:1270 IU/ml

Normal results of: Biotinidase enzyme assay,

Tandem MS, Cranial MRI, Fontanel and Abdominal USG

Skin biopsy: Psoriasiform dermatitis

Ophthalmological examination: Normal findings

DC: Collodion baby, Congenital Erythroderma,

Capillary Malformation, Leiner syndrome

PIC: Eosinophilia and Dysgammaglobulinemia

1-2 years old

E\%: 8.1, IgE:1780 IU/ml

FIPILI/ PDGFRA Mutation: Negative

Skin prick test: Positive reaction for hazelnut and egg white

DC: Atopic dermatitis, PIC: HIES? OS?

\section{2-4 years old}

E\%:15, IgE:2750 IU/ml and E\%:11.2, IgE:2690 IU/ $\mathrm{ml}$

Phadiatop Test: Elevated Serum Specific IgE for hazelnut and egg white

Anti-gliadin IgA: Negative, Anti-endomysial IgA:

Negative

DC: Atopic dermatitis, PIC: HIES? OS?

4 years old to the present

E\%:10.9, IgE:3420 IU/ml and E\%:7.1, IgE:2700

$\mathrm{IU} / \mathrm{ml}$

SPINK5 mutation: Positive

DC: NS, PIC: NS

E: Eosinophil; DC: Dermatological consultation; PIC: Pediatric Immunology consultation; HIES: Hyper IgE syndrome; OS: Omenn syndrome; NS: Netherton syndrome

members refused genetic testing, and we did not obtain a skin sample to extract RNA. Sanger sequencing was applied to SPINK5 (ENST00000359874.7, NM_001127698.1), which revealed a homozygous mutation c. $2112+2 \mathrm{~T}>\mathrm{A}$ in the splice region of intron 22. Following molecular confirmation of the diagnosis of NS, we administered acitretin $0.5 \mathrm{mg} / \mathrm{kg} /$ day and topical moisturizers. Figs. $2 \mathrm{C}, 2 \mathrm{D}$, and $3 \mathrm{~B}$ show the significant beneficial effect of acitretin after one year.

All authors declare their consent for publication of the manuscript. An informed consent was received from the parents of the patient to publish photographs. 

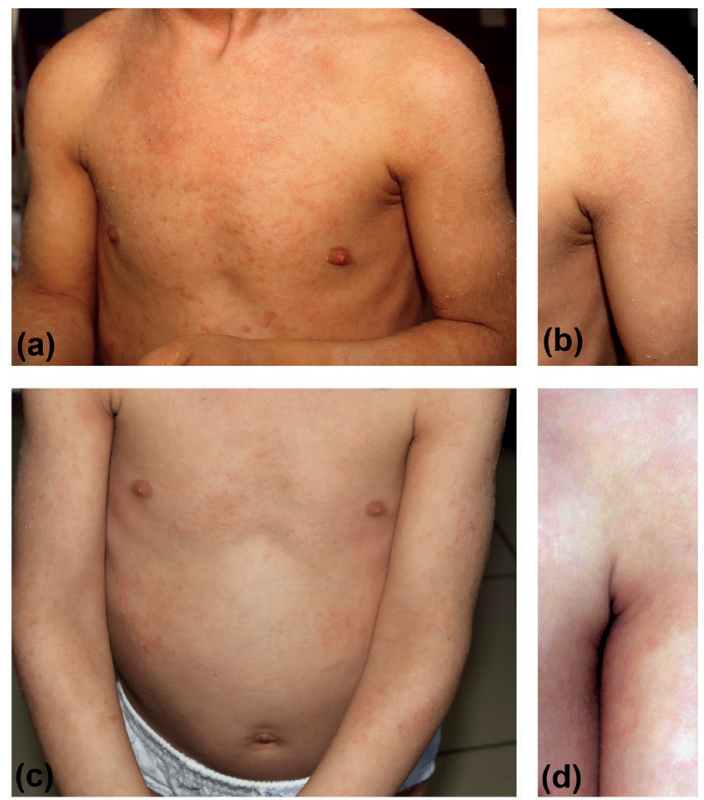

Figs. 2a. Diffuse erythematous and scaly patches (ichthyosis linearis circumflexa) on both the trunk and arms prior to acitretin treatment.

2b. Diffuse erythematous and scaly patches on both the left shoulder and arm prior to acitretin treatment.

2c. The same regions as in Figure 3A, after acitretin treatment.

2d. The same regions as in Figure 3B, after acitretin treatment.

\section{Discussion}

The mutation (c. $2112+2 \mathrm{~T}>\mathrm{A})$ was not previously described and is neither included in the Netherton registry SPINK5 base ${ }^{4}$ nor in other data bases, including the 1000 Genomes Project, the Exome Aggregation Consortium, and the NHLBI Exome Sequencing Project. Because it affects the highly conserved second position of the splice donor site of intron 22, a deleterious effect can be expected. RNA samples from both the boy and his parents were unfortunately unavailable for further assessment of the pathologic mechanism. However, the Mutation Taster algorithm predicted this mutation as "disease causing," which suggests that it may represent a furtherthus far unknown-mutation underlying NS. Moreover, two other mutations that affect the splice donor site of intron 22 were described previously in families from German/
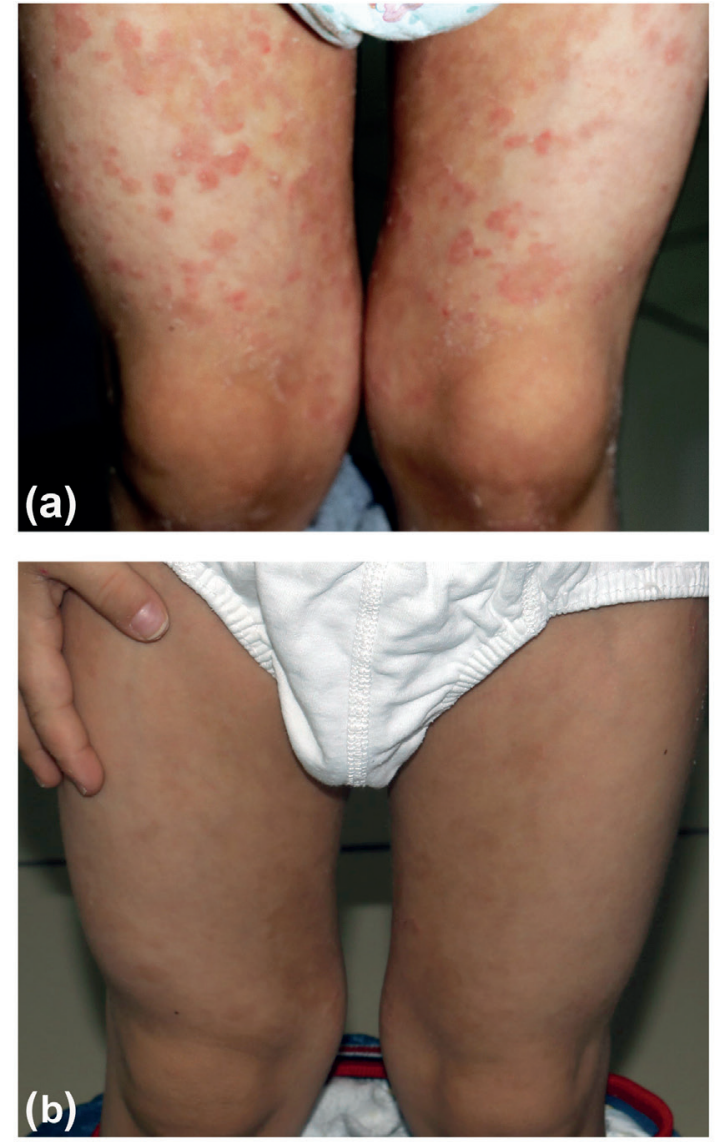

Figs. 3a. Diffuse erythematous and scaly patches on the thighs prior to acitretin treatment.

$3 \mathrm{~b}$. The same regions after acitretin treatment.

Irish and Argentinian descent, respectively: c. 2108 2117del10 and c. $2112+1 \mathrm{G}>\mathrm{A}^{5}{ }^{5}$ However, the Mutation Taster algorithm predicted this mutation as "disease causing," which suggests that it may represent a furtherthus far unknown-mutation underlying NS as it is likely to disturb normal splicing. In total, 84 different mutations of SPINK5 have been registered in the Human Genome Mutation Database. Sarri et al. ${ }^{6}$ investigated 80 mutations that were previously identified in 172 patients from 144 families and demonstrated a wide variety of clinical phenotypes that are associated with these mutations.

HIES includes other autoimmune disorders and musculoskeletal system anomalies that have not been described in NS. Perivascular dermatitis in HIES is different frompsoriasiform dermatitis in NS. Omenn Syndrome exhibits the absence of $\mathrm{B}$ cells and anomalous $\mathrm{T}$ cell 
clones, which are not present in NS., ${ }^{2,3}$ Peeling skin syndrome, type B (PSS-B) shows similar features to NS; however, the clinical outcome is less severe, and the dermatopathology reveals perivascular dermatitis and subcorneal acantholysiS., ${ }^{2,5}$ Severe dermatitis, multiple allergies, metabolic wasting syndrome (SAM) syndrome consists of metabolic wasting, gastroesophageal reflux, and minor cardiac defects, which are not compatible with NS. ${ }^{7}$ Enteropathy, hypoalbuminemia, and urinary extraction of glycine, histidine, and serine may be seen in NS. ${ }^{2,3}$ Metabolic tests, including blood levels of biotinidase enzyme, were conducted on our patient, and the results were normal (Table I). Wiskott-Aldrich syndrome includes thrombocytopenia and normal/low B and $\mathrm{T}$ cell levels. ${ }^{7}$

ILC is described as erythematous patches with two distinct features: It represents typically double-edged scales at the periphery of the lesions and it is migratory. ILC is rarely an isolated clinical manifestation; it is mostly included in NS with congenital ichthyosiform erythroderma, trichorrhexis invaginata (TI), and atopic manifestations. ${ }^{8,9}$ We examined ILC in our patient (Figs. 3a and 3b), and his parents described a migratory appearance of the lesions. Guerra et al. ${ }^{10}$ described two cases of ILC without congenital erythroderma, trichorrhexis invaginata, and symptoms of atopy and claimed ILC may be an isolated sign of NS. Hair anomalies, such as shorth and brittle hair and trichorrhexis invaginata, were absent in our patient, which highlighted the clinical heterogeneity of NS.

NS can represent urticaria/angioedema. ${ }^{1}$ Attacks of urticaria/angioedema were reported in the medical files of our patient. Hazelnut and egg white allergies were defined via laboratory methods, which are summarized in Table I. In addition, we examined eczema in our patient during follow-up visits. Flare-ups of eczema and the need to use topical corticosteroids decreased after acitretin therapy. We found that both ILC and flare-ups of eczema improved during acitretin therapy; however, attacks of urticaria/angioedema did not. We did further consultations with the Department of Pediatric Nutrition and Metabolism, in which a low percentile of both height and weight were found. Whole blood counts, including liver enzymes and blood lipid analyses, were assessed every three months, and no negative effect of acitretin was found.

Our case highlights that a genetic test can be critical for identifying NS. We cannot conclude that this is the definite disease-causing variant. Further investigations like the family genetic testing, and RNA testing are necessary to determine the variant as a mutation. The finding of underlying mutations contributes to the understanding of NS and is instrumental in indicating a specific therapy. Notably, treatment with acitretin has significantly improved both the ILC and eczema in our patient.

\section{REFERENCES}

1. Chavanas S, Bodemer C, Rochat A, et al. Mutations in SPINK5, encoding a serine protease inhibitor, cause Netherton syndrome. Nat Genet 2000; 25: 141-142.

2. Arjona Aguilera C, Albarrán Planelles C, Tercedor Sánchez J. Differential diagnosis of genetic disorders associated with moderate to severe refractory eczema and elevated immunoglobulin E. Actas Dermosifiliogr 2016; 107: 116-124.

3. Williams KW, Milner JD, Freeman AF. Eosinophilia associated with disorders of immune deficiency or immune dysregulation. Immunol Allergy Clin North Am 2015; 35: 523-544.

4. Piirilä H, Väliaho J, Vihinen M. Immunodeficiency mutation databases (IDbases). Hum Mutat 2006; 27: 1200-1208.

5. Sprecher E, Chavanas S, DiGiovanna JJ, et al. The spectrum of pathogenic mutations in SPINK5 in 19 families with Netherton syndrome: implications for mutation detection and first case of prenatal diagnosis. J Invest Dermatol 2001; 117: 179-187.

6. Sarri CA, Roussaki-Schulze A, Vasilopoulos Y, et al. Netherton syndrome: A genotype-phenotype review. Mol Diagn Ther 2017; 21: 137-152.

7. Hernández-Martín A, González-Sarmiento R. Recent advances in congenital ichthyoses. Curr Opin Pediatr 2015; 27: 473-479.

8. Suga Y, Tsuboi R, Hashimoto Y, Yoshiike T, Ogawa H. A case of ichthyosis linearis circumflexa successfully treated with topical tacrolimus. J Am Acad Dermatol 2000; 42: 520-522.

9. Kumar P, Gharami RC, Mondal A, Ghosh K. Ichthyosis linearis circumflexa. Indian Pediatr 2010; 47: 1052.

10. Guerra L, Fortugno P, Pedicelli C, et al. Ichthyosis linearis circumflexa as the only clinical manifestation of Netherton syndrome. Acta Derm Venereol 2015; 95: 720-724. 